ARTICLE

DOI: $10.1038 / s 41467-018-07377-6$

\title{
Double dative bond between divalent carbon(0) and uranium
}

Wei Su${ }^{1}$, Sudip Pan (10 ${ }^{2}$, Xiong Sun ${ }^{1}$, Shuao Wang ${ }^{3}$, Lili Zhao ${ }^{2}{ }^{2}$, Gernot Frenking ${ }^{2,4,5}$ \& Congqing Zhu (iD ${ }^{1}$

Dative bonds between $p$ - and $d$-block atoms are common but species containing a double dative bond, which donate two-electron pairs to the same acceptor, are far less common. The synthesis of complexes between $\mathrm{UCl}_{4}$ and carbodiphosphoranes (CDP), which formally possess double dative bonds $\mathrm{Cl}_{4} \mathrm{U} \leftleftarrows C D P$, is reported in this paper. Single-crystal $\mathrm{X}$-ray diffraction shows that the uranium-carbon distances are in the range of bond lengths for uranium-carbon double bonds. A bonding analysis suggests that the molecules are uranium -carbone complexes featuring divalent carbon(0) ligands rather than uranium-carbene species. The complexes represent rare examples with a double dative bond in $f$-block chemistry. Our study not only introduces the concept of double dative bonds between carbones and $f$-block elements but also opens an avenue for the construction of other complexes with double dative bonds, thus providing new opportunities for the applications of $f$-block compounds.

\footnotetext{
${ }^{1}$ State Key Laboratory of Coordination Chemistry, Jiangsu Key Laboratory of Advanced Organic Materials, School of Chemistry and Chemical Engineering Nanjing University, 210023 Nanjing, China. ${ }^{2}$ Institute of Advanced Synthesis, School of Chemistry and Molecular Engineering, Jiangsu National Synergetic Innovation Center for Advanced Materials, Nanjing Tech University, 211816 Nanjing, China. ${ }^{3}$ State Key Laboratory of Radiation Medicine and Protection, School for Radiological and interdisciplinary Sciences (RAD-X) and Collaborative Innovation Center of Radiation Medicine of Jiangsu Higher Education Institutions, Soochow University, 199 Ren'ai Road, 215123 Suzhou, China. ${ }^{4}$ Fachbereich Chemie, Philipps-Universität Marburg, Hans-Meerwein-Straße 4, 35032 Marburg, Germany. ${ }^{5}$ Donostia International Physics Center (DIPC), P.K. 1072, 20080 Donostia, Euskadi, Spain. Correspondence and requests for materials should be addressed to L.Z. (email: ias_Ilzhao@njtech.edu.cn) or to G.F. (email: frenking@chemie.uni-marburg.de)

or to C.Z. (email: zcq@nju.edu.cn)
} 
U nderstanding the nature of chemical bonding is of great importance, especially for the bonding of carbon with other elements ${ }^{1-4}$. Usually, carbon uses all of its four valence electrons to form stable tetravalent carbon(IV) species. Since the first stable singlet carbene at room temperature was isolated by Bertrand and coworkers ${ }^{3}$, divalent carbon(II) chemistry, such as $\mathrm{N}$-heterocyclic carbene (NHC) ${ }^{6-9}$ and cyclic (alkyl) (amino) carbenes ${ }^{10-13}$, has flourished. Carbenes, $\mathrm{CR}_{2}$, which contain one lone electron pair, can coordinate to numerous main group atoms and $d$-block or even $f$-block elements forming stable species via a single dative bond. If all four carbon valence electrons are retained as two-electron pairs, divalent carbon $(0)$ compounds $\mathrm{CL}_{2}$ ("carbones") are formed ${ }^{14-25}$. Thus, the fundamental difference between a carbene $\mathrm{CR}_{2}$ and a carbone $\mathrm{CL}_{2}$ is the number of electron lone-pairs that may serve as donors: carbenes are two-electron (single lone-pair) donors while carbones are four-electron (double lone-pair) donors. Although the first divalent carbon $(0)$ species $^{9}$, carbodiphosphorane (CDP), was reported already in 1961, the actual bonding situation was not fully understood until a theoretical study appeared in 2006 by Frenking and coworkers ${ }^{27-29}$. They showed that CDP can be considered as two phosphine ligands coordinated to a carbon atom in the excited ${ }^{1} \mathrm{D}$ state with two lone electron pairs, which remain available for both $\sigma$ - and $\pi$-donation simultaneously ${ }^{13}$. There are numerous complexes in which carbones bind to two acceptors $^{31-33}$. In contrast, examples containing a double dative bond, which donates two lone electron pairs to the same acceptor are $\operatorname{rare}^{34-37}$. A comparative experimental work showed that carbenes and carbones exhibit distinctively different complexation behavior due to the varying number of lone-pair orbitals ${ }^{32}$. The chemistry of divalent carbon $(0)$ has been focused mainly on main group compounds and transition metal element complexes. The bonding motif between divalent carbon $(0)$ and $f$-block elements has received less attention. Since uranium plays fascinating roles in energy and catalysis research ${ }^{38-43}$, it is important to isolate species containing a new uranium-carbon bond, which could provide a deeper understanding of the nature of chemical bonding between main group elements and $f$-block metals.

In this paper, we report a set of complexes that possess a double dative bond between carbon and uranium. The complexes were obtained by the reaction of different CDPs with uranium tetrachloride $\left(\mathrm{UCl}_{4}\right)$. Single-crystal X-ray diffraction reveals that the molecules possess rather short uranium-carbon bonds. Density functional theory (DFT) calculations confirm the double dative bond feature between carbon and uranium. The results of this study could be useful for the design of other compounds containing $f$-block atoms that exhibit double dative bonds between the metal and carbone ligands, and could provide new opportunities for the applications of $f$-block elements in catalysis or in the activation of small molecules.

\section{Results}

Synthesis and characterization. We first designed and prepared a new tridentate CDP precursor, 1-( $\left.\mathbf{P F}_{\mathbf{6}}\right)_{2}$, with two 2-(pyridyl) diphenylphosphine units (see Supplementary Methods). The corresponding $\mathrm{CDP}-\mathrm{UCl}_{4}$ adduct (2) was readily synthesized by the deprotonation of compound $\mathbf{1}-\left(\mathbf{P F}_{\mathbf{6}}\right)_{\mathbf{2}}$ with NaHMDS and subsequent reaction in situ of the resulting CDP with $\mathrm{UCl}_{4}$ (Fig. 1a). Complex 2 was isolated in $66 \%$ yield as yellow-green crystals after recrystallization at $-35^{\circ} \mathrm{C}$ overnight. The crystalline form of $\mathbf{2}$ shows low solubility in most organic solvents and easily decomposes to the free ligand. Consequently, the ${ }^{31} \mathrm{P}$ nuclear magnetic resonance (NMR) spectrum shows two equivalent phosphine signals at -215.87 ppm for complex 2 along with the signal for 1 (Supplementary Fig. 1). The ${ }^{1} \mathrm{H}$ NMR spectrum of a

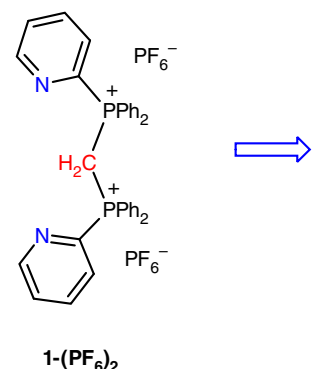

1- $\left(\mathrm{PF}_{6}\right)_{2}$
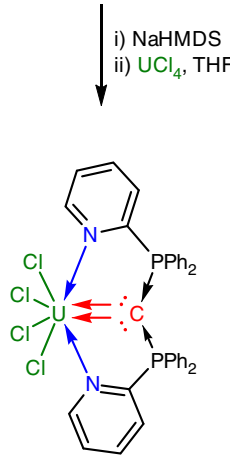

2 b
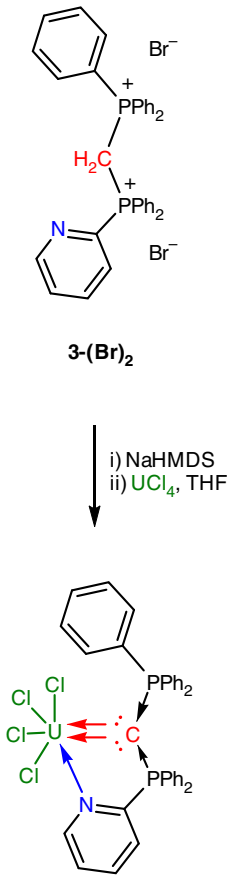

4
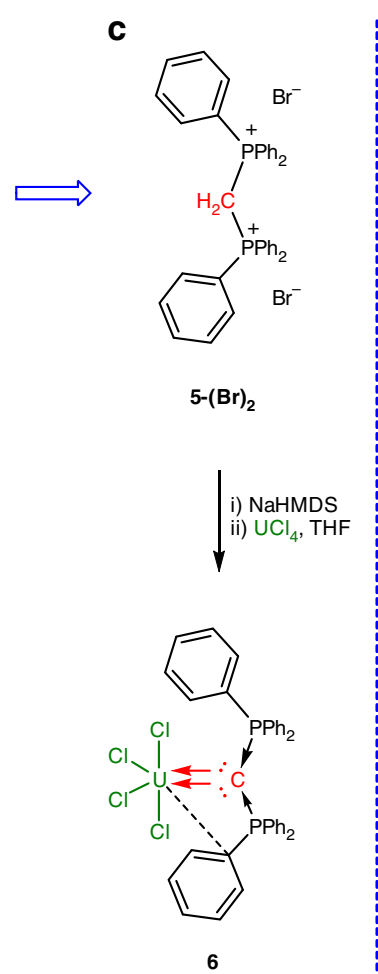

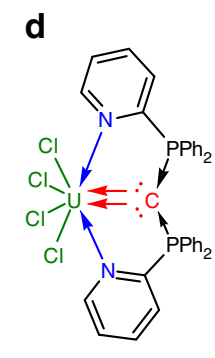

2a

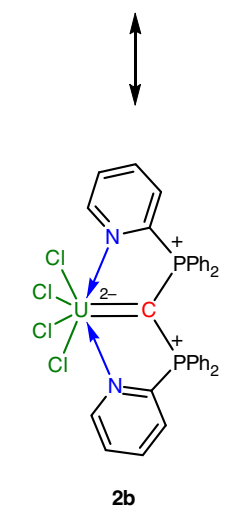

Fig. 1 Synthesis of $C D P-U C l_{4}$ adducts. Synthesis of double dative bond adducts $\mathbf{2}(\mathbf{a}), \mathbf{4}(\mathbf{b})$, and $\mathbf{6}$ (c) employ tridentate, bidentate, and monodentate CDP precursors 1-( $\mathbf{P} \mathbf{F}_{\mathbf{6}} \mathbf{2}_{\mathbf{2}}, \mathbf{3 -}-(\mathbf{B r})_{\mathbf{2}}$, and $\left.\mathbf{5 - ( B r}\right)_{\mathbf{2}}$, respectively. $\mathbf{d}$ Two major resonance structures of complex $\mathbf{2}$ with a double dative bond (2a) and with an electron-sharing $\mathrm{U}=\mathrm{C}$ double bond $(\mathbf{2} \mathbf{b})$ 
a

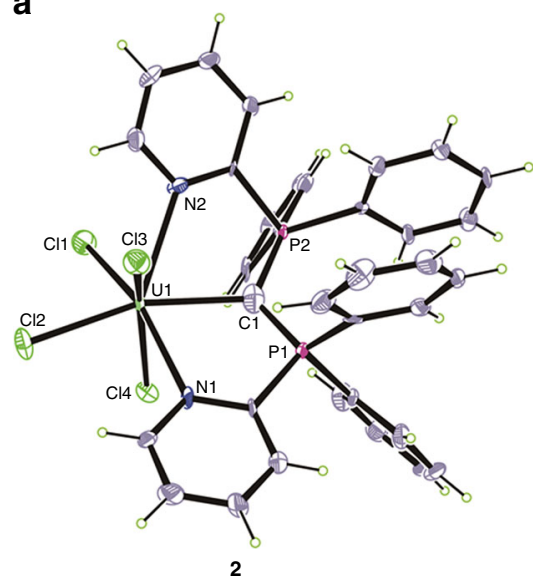

b

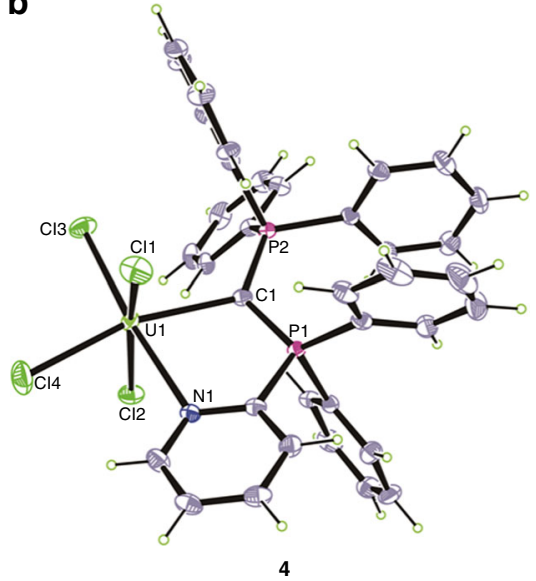

C

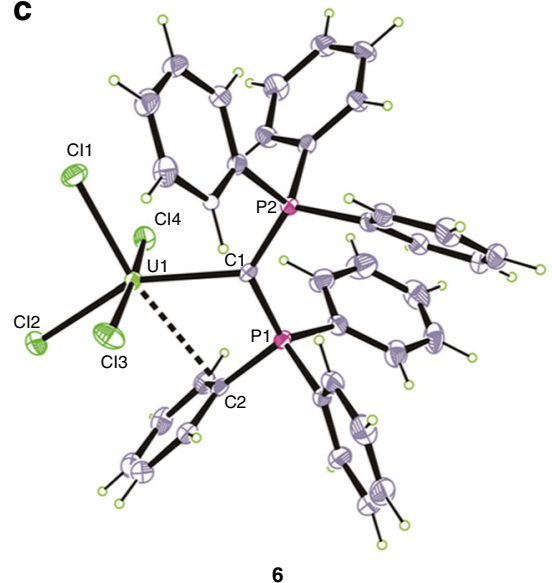

Fig. 2 Molecular structures of CDP-UCl ${ }_{4}$ adducts 2, 4, and 6. a-c Solid-state structures of $\mathbf{2}$ (a), $\mathbf{4}$ (b), and $\mathbf{6}$ (c) by X-ray crystallography with $50 \%$ probability ellipsoids. Solvent molecules are omitted for clarity. Selected experimental [calculated] bond distances ( $\AA$ ) and angles (deg) for 2: U1-C1 2.471 (7) [2.425], U1-N1 2.625(5) [2.612], U1-N2 2.582(6) [2.577], U1-Cl1 2.649(2) [2.612], U1-Cl2 2.645(2) [2.577], U1-Cl3 2.644(2) [2.663], U1-Cl4 2.655 (2) [2.651], C1-P1 1.701(7) [1.680], C1-P2 1.690(6) [1.684], P1-C1-P2 121.3(4) [123.0]. For 4: U1-C1 2.461(5) [2.461], U1-N1 2.537(4) [2.530], U1-Cl1 2.598(2) [2.617], U1-Cl2 2.570(2) [2.615], U1-Cl3 2.592(2) [2.566], U1-Cl4 2.622(2) [2.570], C1-P1 1.711(5) [1.692], C1-P2 1.699(5) [1.711], P1-C1-P2 120.6(3) [117.7]. For 6: U1-C1 2.411(3) [2.436], U1-Cl1 2.550(1) [2.544], U1-Cl2 2.604(1) [2.554], U1-Cl3 2.594(1) [2.603], U1-Cl4 2.634(1) [2.609], C1-P1 1.705(3) [1.699], C1-P2 1.719(3) [1.710], P1-C1-P2 125.05(16) [121.9]. Uranium, yellow-green; phosphorus, violet red; nitrogen, blue; chlorine, green; carbon, gray

complex 2 exhibits a broad range of peaks from +14.19 to -41.54 ppm (Supplementary Fig. 2).

The solid-state structure of $\mathbf{2}$ was confirmed by an analysis of its X-ray diffraction pattern (Fig. 2a). The uranium atom adopts a twisted pentagonal bipyramidal geometry in which both pyridyl ligands are coordinated to the uranium center forming two fivemembered rings. The average $\mathrm{U}-\mathrm{N}$ and $\mathrm{U}-\mathrm{Cl}$ bond lengths are 2.604(5) $\AA$ and 2.648(2) $\AA$, respectively. The bond length of $\mathrm{U} 1-\mathrm{C} 1$ was found to be 2.471(7) $\AA$, which is obviously shorter than the $\mathrm{U}-\mathrm{C}$ bond distances found in the adducts of NHC with uranium (2.573 $\AA-2.788 \AA$ ) (based on a search of the Cambridge Structural Database, CSD version 5.39 (updates 2017)). This $\mathrm{U}-\mathrm{C}$ bond length in complex $2(2.471(7) \AA)$ is in the range of $\mathrm{U}(\mathrm{IV})=\mathrm{C}$ double bond distances $(2.310 \AA-2.578 \AA)$ in complexes with bis(iminophosphorano)methandiide as ligands (based on a search of the Cambridge Structural Database, CSD version 5.39 (updates 2017)). An alternative description of 2 with a double bond between uranium and carbon is provided by the resonance structure $\mathbf{2 b}$ (Fig. 1d). This depicts a carbon(IV) atom with an electron-sharing $\mathrm{U}=\mathrm{C}$ double bond in which uranium has a formal charge of -2 . Analysis of the bonding suggests that $2 \mathbf{a}$ is the dominant resonance form contributing to the bonding situation.

The short $\mathrm{U}-\mathrm{C}$ bond in complex 2 prompted us to appraise uranium-carbon multiple bond chemistry ${ }^{44-46}$. The first uranium carbene was reported in 1981, and was stabilized by a phosphorus substituent ${ }^{29}$. Subsequently, various species containing a uranium carbon double bond with one or two phosphorus substituents on the carbene carbon were reported ${ }^{48-63}$. Interestingly, most of the reported uranium carbenes utilize tridentate chelating methanediide ligands ${ }^{4-64}$. Indeed, some of the uranium carbene species in the literature might be considered to contain non-negligible carbone character in their bonding $49,56,54$. In addition, species containing a $\mathrm{U}-\mathrm{C}$ double bond, triple bond, or even quadruple bond were identified by matrix infrared spectroscopy and/or relativistic density functional calculations $^{64-69}$. Very recently, a diuranium carbide cluster $(\mathrm{U}=\mathrm{C}=\mathrm{U})$ was also stabilized inside a $\mathrm{C}_{80}$ fullerene cage ${ }^{50}$.
In order to investigate the effect of CDP ligands on the bonding between divalent carbon $(0)$ and uranium, analogous bidentate and monodentate CDP precursors $3-(\mathbf{B r})_{2}$ and $\mathbf{5}-(\mathbf{B r})_{2}$ were synthesized. As shown in Fig. $1 \mathrm{~b}, \mathrm{c}$, treatment of $\mathrm{UCl}_{4}$ with the CDP ligands, generated by the in situ deprotonation of $3-(\mathbf{B r})_{2}$ and 5-(Br $)_{2}$ with NaHMDS in THF, resulted in the formation of CDP- $\mathrm{UCl}_{4}$ adducts 4 and $\mathbf{6}$ in $52 \%$ and $59 \%$ crystalline yields, respectively, after recrystallization at $-35^{\circ} \mathrm{C}$. Both crystals of complexes $\mathbf{4}$ and $\mathbf{6}$ totally decomposed after re-dissolved in common solvents. Fortunately, the ${ }^{31} \mathrm{P}$ NMR spectrum of complex 4 was recorded from the in situ reaction solutions. The signals of two nonequivalent phosphorus centers in this species were observed at -125.51 and -155.13 ppm (Supplementary Fig. 3). Complex 6 represents an unusual non-chelated species with a double dative bond between carbon and an $f$-block element compared with reported work $47,48,67$, but its stability is significantly lower than that of chelating complexes $\mathbf{2}$ and $\mathbf{4}$.

The crystal structure of complex 4 (Fig. 2b) reveals a $\mathrm{U} 1-\mathrm{C} 1$ bond distance of 2.461(5) $\AA$, which compares well with that found in complex 2. The bond distances of U1-N1 (2.537(4) $\AA$ ) and average $\mathrm{U}-\mathrm{Cl}(2.595(2) \AA)$ in $\mathbf{4}$ were slightly shorter than those observed in complex 2 . The pyridyl unit coordinates with uranium center to form a five-membered ring, generating a distorted octahedral configuration. An X-ray study of 6 showed the uranium to have a distorted octahedral structure, concerning a weak interaction between the uranium center and a phenyl carbon atom, with a U1-C1 bond length of 2.411(3) $\AA$ (Fig. 2c). The bond lengths of $\mathrm{U} 1-\mathrm{C} 1$ in all these complexes are obviously shorter than the U-C distances $(2.573 \AA-2.788 \AA)$ typically observed in NHC-uranium adducts. These data suggest that the bond between the divalent carbon( 0 ) of CDP and the uranium is a multiple bond.

The recording of reliable absorption spectra of these complexes is prevented by their poor stability in solution. Fourier-transform infrared (FT-IR) spectra demonstrated bands at $690 \mathrm{~cm}^{-1}$ for 2, $687 \mathrm{~cm}^{-1}$ for 4 , and $686 \mathrm{~cm}^{-1}$ for 6 , respectively. These data match excellently with those computed for U-C stretching $(674$ $\mathrm{cm}^{-1}$ for $2,673 \mathrm{~cm}^{-1}$ for 4 , and $671 \mathrm{~cm}^{-1}$ for $\mathbf{6}$, Supplementary 


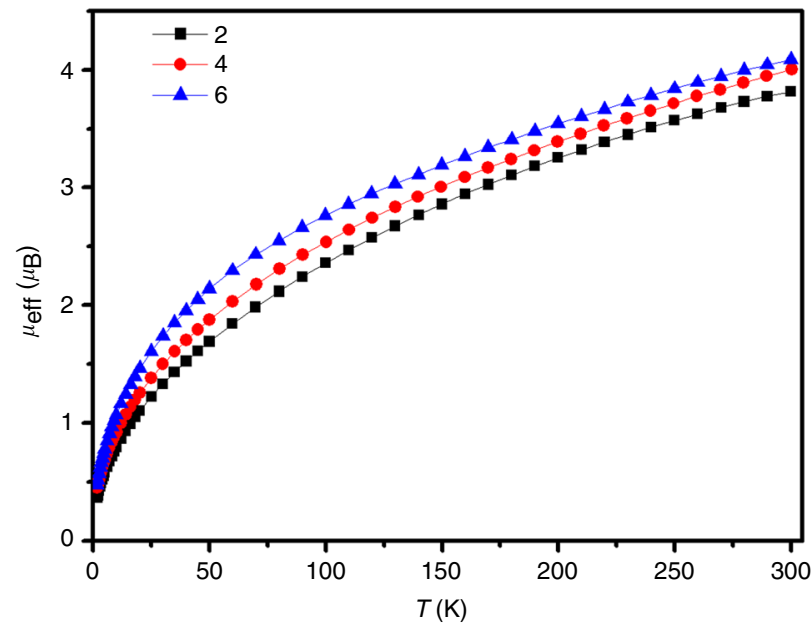

Fig. 3 Magnetic characterization data. Variable-temperature SQUID magnetization data of complex $\mathbf{2}$ (black square), complex $\mathbf{4}$ (red circle), and complex 6 (blue triangle) at $0.1 \mathrm{~T}$. Lines are a guide to the eye only

Table 3), suggesting the intrinsic double dative bond between uranium center and carbone carbon atom. Furthermore, variabletemperature magnetic measurements were used to characterize these species in the solid state. The magnetic moment of $\mathbf{2}$ at 300 $\mathrm{K}$ is approximately $3.81 \mu_{\mathrm{B}}$, which is slightly higher than the expected value $\left(3.58 \mu_{\mathrm{B}}\right)$ for the ${ }^{3} \mathrm{H}_{4}$ ground state of $f^{2}$ uranium (IV). Upon cooling, the magnetic moments decrease smoothly to $0.37 \mu_{\mathrm{B}}$ at $1.8 \mathrm{~K}$ and tending to zero (Fig. 3 and Supplementary Figs. 4-6). The magnetic moments and trends of $\mathbf{4}$ and $\mathbf{6}$ are similar to those of 2 , which are consistent with uranium(IV) centers in those species. The uranium(IV) centers in these complexes were further confirmed by the field-dependent magnetization data collected at $1.8 \mathrm{~K}$ (Supplementary Figs. 7-9).

Theoretical studies. DFT calculations were performed to elucidate the bonding situation in $\mathbf{2 , 4}$, and $\mathbf{6}$. The geometries of these species were optimized at the BP86-D3(BJ)/def2-TZVPP/Stuttgart RSC ECP level using scalar-relativistic effective core potentials for uranium (see Supplementary Methods). Calculations at various spin states suggest that 2, 4, and $\mathbf{6}$ have an electronic triplet ground state. Calculated structures at the electronic singlet and quintet state are much higher in energy (Supplementary Fig. 10). The calculated bond lengths and angles of the three complexes are in good agreement with the experimental data (Fig. 2). The differences between theory and experiment are within the range of solid-state effects and the accuracy of the methods.

We calculated the bond strength of the uranium-carbon bonds in $\mathbf{2 , 4}$, and $\mathbf{6}$. The theoretically predicted bond dissociation energies (BDEs) suggest (reactions $1-3$ ) that the introduction of pyridine substituents for phenyl enhances the bond strength, which can be explained with the contribution of the $\mathrm{N} \rightarrow \mathrm{U}$ dative bonds to the uranium-CDP bonds. The calculated BDE for 6 $\left(D_{e}=70.8 \mathrm{kcal} \mathrm{mol}^{-1}\right)$ indicates that the unsupported $\mathrm{U} \leftleftarrows \mathrm{C}$ double donor bond is rather strong. Hayton and coworkers ${ }^{44}$ previously reported that the upper limit for the $\mathrm{U}=\mathrm{C} B \mathrm{BDE}$ value in the related system is $90 \mathrm{kcal} \mathrm{mol}^{-1}$, which is in good agreement with our results. Nonetheless, the values are significantly lower than the BDE value of $\mathrm{Ta}=\mathrm{C}$ (i.e., $126 \mathrm{kcal} \mathrm{mol}^{-1}$ ) in $\mathrm{Ta}=\mathrm{CHR}$ $\left(\mathrm{CH}_{2} \mathrm{R}\right)_{3}\left(\mathrm{R}=\mathrm{SiMe}_{3}\right)^{52}$. This shows the difference in bond strength between bonds involving an actinide and a formal carbone and those with a transition metal and a formal
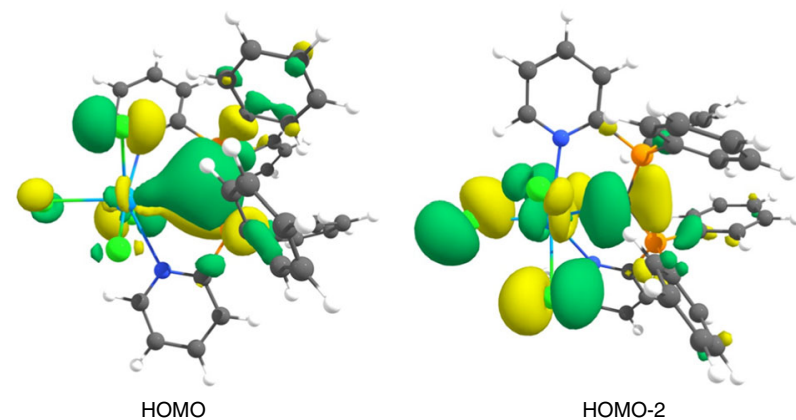

Fig. 4 Shape of the HOMO and the HOMO-2 of complex 2 . The isosurface value is 0.03 e $\AA^{-3}$

Table 1 EDA-NOCV results by using the triplet $(\mathrm{T}) \mathrm{UCl}_{4}$ and singlet (S) CDP fragments

\begin{tabular}{|c|c|c|c|}
\hline & $\begin{array}{l}2 \\
\mathrm{UCl}_{4}(\mathrm{~T})+\mathrm{CDP} \\
(\mathrm{S})\end{array}$ & $\begin{array}{l}4 \\
\mathrm{UCl} \\
(\mathrm{S})\end{array}$ & $\begin{array}{l}6 \\
\mathrm{UCl}_{4}(\mathrm{~T})+\mathrm{CDP} \\
(\mathrm{S})\end{array}$ \\
\hline $\begin{array}{l}\Delta E_{\text {int }}{ }^{\mathrm{a}} \\
\Delta E_{\text {Pauli }} \\
\Delta E_{\text {disp }}{ }^{\mathrm{b}} \\
\Delta E_{\text {elstat }}{ }^{\mathrm{b}} \\
\Delta E_{\text {orb }}{ }^{\mathrm{b}} \\
\Delta E_{\text {orb1 }}{ }^{\mathrm{c}} \\
\Delta E_{\text {orb2 }}{ }^{\mathrm{c}} \\
\Delta E_{\text {orb3 }}{ }^{\mathrm{c}} \\
\Delta E_{\text {orb4 }}{ }^{\mathrm{c}} \\
\Delta E_{\text {orb(rest) }}{ }^{\mathrm{c}}\end{array}$ & $\begin{array}{l}-133.4 \\
233.0 \\
-37.6(10.3 \%) \\
-186.2(50.8 \%) \\
-142.6(38.9 \%) \\
-39.4(27.6 \%) \\
-17.1(12.0 \%) \\
-17.1(12.0 \%) \\
-14.2(10.0 \%) \\
-54.8(38.5 \%)\end{array}$ & $\begin{array}{l}-121.4 \\
187.9 \\
-35.4(11.4 \%) \\
-154.3(49.9 \%) \\
-119.5(38.6 \%) \\
-37.7(31.5 \%) \\
-13.5(11.3 \%) \\
-15.2(12.7 \%) \\
-11.2(9.4 \%) \\
-41.9(35.0 \%)\end{array}$ & $\begin{array}{l}-91.9 \\
159.2 \\
-37.5(14.9 \%) \\
-125.8(50.1 \%) \\
-87.9(35.0 \%) \\
-27.9(31.7 \%) \\
-13.3(15.1 \%) \\
-8.6(9.8 \%) \\
-6.5(7.4 \%) \\
-31.6(35.9 \%)\end{array}$ \\
\hline \multicolumn{4}{|c|}{$\begin{array}{l}\text { aEnergy values are given in } \mathrm{kcal} \mathrm{mol}^{-1} \\
\text { bThe values in parentheses show the contribution towards the total attractive interaction } \\
\Delta E_{\text {elstat }}+\Delta E_{\text {orb }}+\Delta E_{\text {disp }} \\
\text { cThe values in parentheses show the contribution towards the total orbital interaction, } \Delta E_{\text {orb }}\end{array}$} \\
\hline
\end{tabular}

alkylidene.

$$
\begin{gathered}
\mathbf{2} \rightarrow \mathrm{UCl}_{4}\left({ }^{3} \mathrm{~A}\right)+\mathrm{C}\left(\mathrm{PPh}_{2} \mathrm{Py}\right)_{2} D_{e}=91.5 \mathrm{kcal} \mathrm{mol}^{-1}, \\
\mathbf{4} \rightarrow \mathrm{UCl}_{4}\left({ }^{3} \mathrm{~A}\right)+\mathrm{C}\left(\mathrm{PPh}_{2} \mathrm{Py}\right)\left(\mathrm{PPh}_{3}\right) D_{e}=82.8 \mathrm{kcal} \mathrm{mol}^{-1}, \\
\mathbf{6} \rightarrow \mathrm{UCl}_{4}\left({ }^{3} \mathrm{~A}\right)+\mathrm{C}\left(\mathrm{PPh}_{3}\right)_{2} D_{e}=70.8 \mathrm{kcal} \mathrm{mol}^{-1}
\end{gathered}
$$

The calculated spin densities in 2, 4, and $\mathbf{6}$ indicate that the unpaired electrons are localized mainly at the uranium atom (Supplementary Fig. 11). This agrees with the shape of the single occupied molecular orbitals (SOMO) and SOMO-1, which are also centered at uranium center (Supplementary Figs. 12-13). Figure 4 shows the highest occupied molecular orbital (HOMO) and HOMO-2 of complex 2 , which may be identified with the $\pi$ and $\sigma$ dative bonds of $\mathrm{Cl}_{4} \leftleftarrows \mathrm{CDP}$. The related MOs of 4 and $\mathbf{6}$ are very similar; they are shown in Supplementary Figs. 12-13.

More detailed information about the nature of the $\mathrm{Cl}_{4} \mathrm{U}-\mathrm{CDP}$ interactions is available from energy decomposition analysis with natural orbitals for chemical valence (EDA-NOCV) calculations of 2, 4, and 6 using the fragments $\left({ }^{3} \mathrm{~A}\right) \mathrm{UCl}_{4}$ and singlet $\mathrm{CDP}$ with their frozen geometries of the complexes as interacting species. Further details about the method are given in Supplementary Methods. Table 1 shows the numerical results.

The intrinsic interactions energies $\Delta E_{\text {int }}$ exhibit the same trend for the uranium-carbon bond strengths as the BDEs $(2>4>6)$. 
a
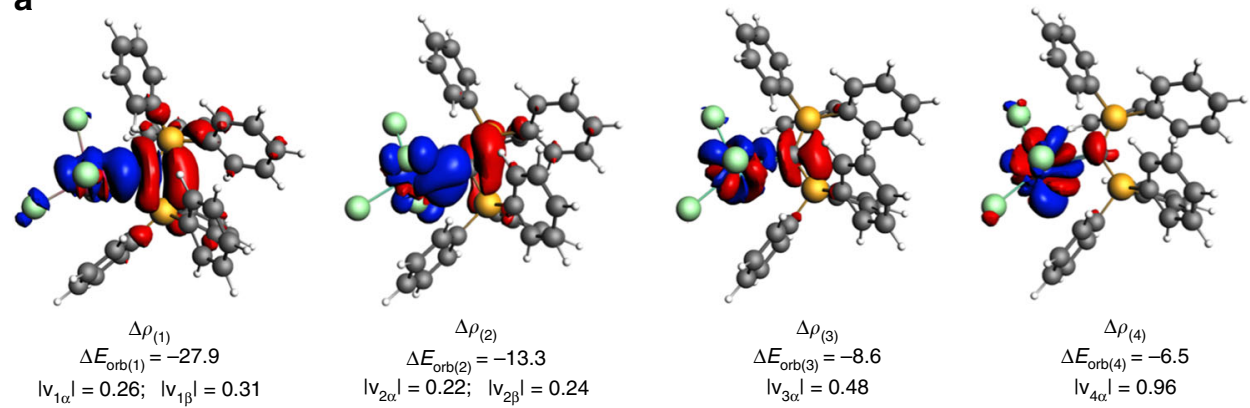

$\mathrm{Cl}_{4} \mathrm{U}\left(\mathrm{C}\left(\mathrm{PPh}_{3}\right)_{3}, 6\right.$
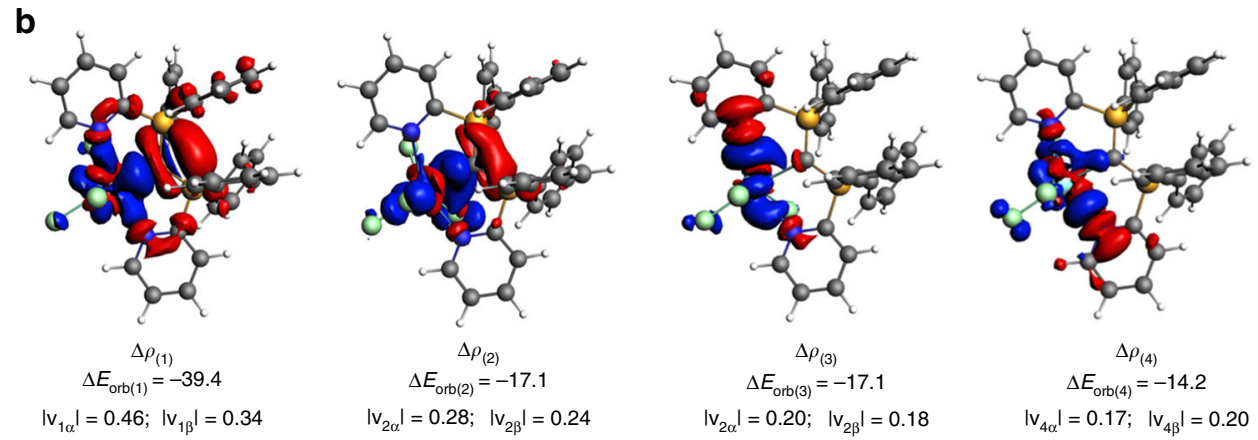

$\mathrm{Cl}_{4} \mathrm{U}\left(\mathrm{C}\left(\mathrm{PPh}_{2} \mathrm{Py}\right)_{2}, 2\right.$

Fig. 5 Plot of the deformation densities $\Delta \rho$ together with the associated interaction energies $\Delta E_{\text {orb }}$. Deformation densities $\Delta \rho_{1-4}$ of the pairwise orbital interactions in $\mathbf{6}$ (a) and $\mathbf{2}(\mathbf{b})$ between $\mathrm{UCl}_{4}$ and the CDP ligand. The energies are in $\mathrm{kcal} \mathrm{mol}^{-1}$. The charge eigenvalues $\nu$ give an estimate of the relative size of the charge migration. The direction of the charge flow is red $\rightarrow$ blue

Table 2 Calculated NBO compositions of the $U-C$ natural orbitals in the complexes 2, 4, and 6

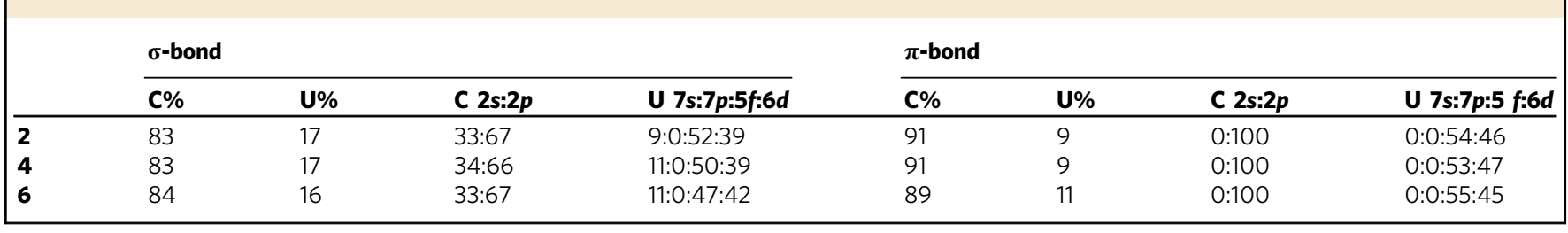

Inspection of the attractive terms indicates that the dispersion make a non-negligible contribution between 10 and $15 \%$ of the total attraction. Furthermore, the uranium-carbon bonds possess a more electrostatic than covalent character as revealed by the relative strength of $\Delta E_{\text {elstat }}$ and $\Delta E_{\text {orb. }}$. The most important information comes from the breakdown of the total orbital (covalent) interactions into pairwise orbital contributions $\Delta E_{\text {orb }}$ (x). The strongest interactions $\Delta E_{\text {orb(1) }}$ and $\Delta E_{\text {orb(2) }}$ are due to the $\sigma$ and $\pi$ components of the double donation $\mathrm{Cl}_{4} \mathrm{U} \leftleftarrows \mathrm{CDP}$. The nature of the orbital interactions comes to the fore when inspecting the shapes of the associated deformation densities $\Delta \rho_{(1)}$ and $\Delta \rho_{(2)}$ in 6, which are shown in Fig. 5a. There is clearly a charge donation from the $\sigma$ and $\pi$ lone-pair electrons on carbon to the uranium atom. The next strongest interactions $\Delta E_{\mathrm{orb}(3)}$ and $\Delta E_{\text {orb(4) }}$ come from the singly occupied orbitals, which involves some further donation from the CDP ligand to uranium. The remaining orbital terms are mainly due to polarization within the fragments.

Figure $5 \mathrm{~b}$ shows the deformation densities $\Delta \rho$ of the four strongest pairwise orbital interactions in complex 2. The stabilization energies $\Delta E_{\mathrm{orb}(1)}$ and $\Delta E_{\mathrm{orb}(2)}$ are again due to the $\sigma$ and $\pi$ components of the double donation $\mathrm{Cl}_{4} \mathrm{U} \leftleftarrows \mathrm{CDP}$. The shape of $\Delta \rho_{(3)}$ and $\Delta \rho_{(4)}$ suggest that $\Delta E_{\text {orb(3) }}$ and $\Delta E_{\text {orb(4) }}$ are associated with the additional $\mathrm{N} \rightarrow \mathrm{U}$ donation of the pyridine ligands, which have a similar strength as the $\mathrm{Cl}_{4} \mathrm{U} \leftarrow \mathrm{CDP} \sigma$ donation. In summary, the EDA-NOCV calculations strongly suggest that the CDP units in $\mathbf{2 , 4}$, and $\mathbf{6}$ serve as double donor ligands toward uranium. A previous study by Liddle and coworkers reported a gallium-uranium complex with a $\mathrm{U} \Leftarrow \mathrm{Ga}$ $\sigma$ and $\pi$ bond ${ }^{53}$. They wrote that " $\pi$ donation by carbene-type fragments may be more widespread than previously recognized". Here we report an example where the ligand is a carbone $\mathrm{CL}_{2}$.

In order to identify the valence atomic orbitals of uranium, which serve as acceptor orbitals for the donor-acceptor interaction with the CDP ligands, we carried out natural bond orbital (NBO) calculations of 2, 4, and 6. Supplementary Fig. 14 shows the shape of two natural bond orbitals that can easily be identified with the $\sigma$ and $\pi$ bonds in $\mathrm{Cl}_{4} \mathrm{U} \leftleftarrows \mathrm{CDP}$. Both bonds are strongly polarized toward the carbon end, which agrees with the findings of Liddle and coworkers in the uranium complexes employing methanediide ligand ${ }^{54}$. Table 2 shows the numerical results of the NBO calculations. Inspection of the hybridization at uranium reveals that the $\sigma$ and $\pi$ acceptor orbitals are mainly composed of $5 f$ and $6 d$ atomic orbitals, with the former being the larger component. Specifically the $\mathrm{U}-\mathrm{C} \sigma$-bond and $\pi$-bonds in 2 are polarized with $83 \%$ and $91 \%$ at the carbon, respectively, which 


\begin{tabular}{|c|c|c|c|c|c|}
\hline Complex & BCPa & $\rho\left(r_{c}\right)^{b}$ & $\nabla^{2} \rho\left(r_{c}\right)^{c}$ & $H\left(r_{c}\right)^{d}$ & $\varepsilon\left(r_{c}\right)^{e}$ \\
\hline \multirow[t]{2}{*}{2} & U-C1 & 0.079 & 0.112 & -0.018 & 0.09 \\
\hline & U-N1 & 0.053 & 0.146 & -0.005 & \\
\hline \multirow[t]{2}{*}{4} & U-N2 & 0.050 & 0.139 & -0.004 & \\
\hline & U-C1 & 0.075 & 0.107 & -0.016 & 0.12 \\
\hline \multirow[t]{3}{*}{6} & U-N & 0.059 & 0.156 & -0.006 & \\
\hline & $\mathrm{U}-\mathrm{C} 1$ & 0.079 & 0.109 & -0.018 & 0.11 \\
\hline & U-C2 & 0.015 & 0.046 & 0.008 & \\
\hline \multicolumn{6}{|c|}{$\begin{array}{l}\text { aThe bond critical points } \\
\text { bThe electron density }\left(\rho\left(r_{c}\right) \text {, au }\right) \\
\text { cLaplacian of electron density }\left(\nabla^{2} \rho\left(r_{c}\right) \text {, au }\right) \\
\text { dTotal electronic energy density }\left(H\left(r_{c}\right) \text {, au }\right) \\
\text { eEllipticity of electron density }\left(\varepsilon\left(r_{c}\right)\right)\end{array}$} \\
\hline
\end{tabular}

strongly supports the notation of carbon as double donor. The uranium part of the $\mathrm{U}-\mathrm{C} \sigma$-bond has $52 \% f$ and $39 \% d$ character. The NBO data for $\mathbf{4}$ and $\mathbf{6}$ are very similar to those of complex $\mathbf{2}$.

The natural charges and bond orders are provided in Supplementary Table 4 where the charge distribution indicates that $0.64(2), 0.52(4)$ and $0.50(6) \mathrm{e}^{-}$are transferred from CDP to $\mathrm{UCl}_{4}$ fragment. Therefore, the ligand behavior of CDP is significantly enhanced in the presence of the two pyridyl rings. The $\mathrm{C} 1$ center still carries a quite large negative charge $(-1.39$ to $-1.43 \mathrm{e}^{-}$) and this might be due to the significant contribution from its dipolar resonance form and/or the electron density accumulation resulting from an electron transfer from $\mathrm{PR}_{3}$ to $\mathrm{C} 1$. In principle, both are responsible in varying degrees. The Nalewajski-Mrozek bond order $(\mathrm{NMBO})^{74-77}$, which represents the combined covalent and ionic bond order, has been previously reported to be very well correlated with the $\mathrm{U}=\mathrm{C}$ bond description. In our study, the $\mathrm{NMBO}$ of 2, 4, and $\mathbf{6}$ was computed as $1.30,1.34$, and 1.41 , respectively, indicating the double bond character therein.

Finally, the results of quantum theory of atoms in molecules (QTAIM) analysis are provided in Table 3. Note that for covalent bonds, $\nabla^{2} \rho\left(r_{\mathrm{c}}\right)$ is usually negative; however, this criterion often fails for the bonds involving heavier elements. This is because $\nabla^{2} \rho$ $\left(r_{c}\right)$ derives from the three curvature values $\left(\lambda_{1}, \lambda_{2}\right.$ and $\left.\lambda_{3}\right)$ where first two terms are negative but $\lambda_{3}$ is positive. For heavier elements, $\lambda_{3}$ term often dominates over the other two terms making the overall $\nabla^{2} \rho\left(r_{c}\right)$ value positive. For these cases, the use of $H\left(r_{\mathrm{c}}\right)$ is recommended which is usually negative for covalent bonds. In the present cases, although $\nabla^{2} \rho\left(r_{c}\right)$ is positive, the corresponding $H\left(r_{\mathrm{c}}\right)$ values are negative for $\mathrm{U}-\mathrm{Cl}$ and $\mathrm{U}-\mathrm{N}$ bonds. Moreover, the $H\left(r_{\mathrm{c}}\right)$ value in the former bond is considerably more negative than that in the latter one, indicating stronger covalent interaction in the former case. The weak interaction of the $\mathrm{U}-\mathrm{C} 2$ (phenyl carbon) bond in $\mathbf{6}$ is reflected from the positive $H\left(r_{\mathrm{c}}\right)$ value (Supplementary Fig. 15). We have also computed ellipticity of electron density $\left(\varepsilon\left(r_{c}\right)\right)$ at the BCP of $\mathrm{U}-\mathrm{C} 1$ bond. In general, for a single bond $(\sigma)$ and triple bond $(\sigma+2 \pi)$ which have cylindrical contours of $\rho$, the corresponding $\varepsilon\left(r_{\mathrm{c}}\right)$ value is approximately zero, whereas for a double bond $(\sigma+\pi)$, because of the asymmetric distribution of $\rho$ in perpendicular to the bond path the $\varepsilon\left(r_{\mathrm{c}}\right)$ value is greater than zero ${ }^{58}$. In the present complexes, the $\varepsilon\left(r_{\mathrm{c}}\right)$ value turned out as 0.09 (2), 0.12 (4) and $0.11(6)$. Although these values reflect slight perturbation from an ideal single bond, they are significantly smaller than the other corresponding values reported for $\mathrm{U}=\mathrm{C}$ bonds in chelated complexes which range from 0.22 to $0.52^{58}$. Therefore, the ellipticity data do not clearly corroborate with the outcome from the NBO and EDA-NOCV analyses. Perhaps, this might be due to the very polarized nature of the double dative bonds.

\section{Discussion}

We have prepared a set of complexes formed between CDP ligands and $\mathrm{UCl}_{4}$, which contain double dative bonds between carbon and uranium. Single-crystal X-ray diffraction analysis revealed a short uranium-carbon bond in these species. Theoretical calculations suggest that the nature of the bond between the carbon of CDP and uranium is a double dative bond. Both of the $\sigma$ and $\pi$ dative bonds in $\mathrm{Cl}_{4} \mathrm{U} \leftleftarrows \mathrm{CDP}$ are strongly polarized toward the carbon. Therefore, the concept of a double dative bond between carbon and $f$-block elements has been proposed. This finding shows that the carbones could serve as an effective ligand for the synthesis of $f$-block species with a double dative bond. These complexes reported herein further strengthen our understanding of the bonding between carbon and uranium, and thus the double dative bond might be a more general bonding motif in $f$-block chemistry. Our further studies will focus on the synthesis of other $f$-block species containing a double dative bond employ carbones as ligands, as well as investigations of their applications in small molecule activation.

\section{Methods}

General considerations. Experiments were performed under an $\mathrm{N}_{2}$ atmosphere using standard Schlenk-line and glove-box techniques. All solvents and reagents were dried and deoxygenated before use, using a solvent purification system. See the Supplementary Methods for detailed experimental procedures, crystallographic (Supplementary Tables 1-2), and computational analyses (Supplementary Dataset and Supplementary Tables 3-4).

Preparation of 2. NaHMDS ( $0.2 \mathrm{~mL}, 2 \mathrm{M}$ in THF, $0.4 \mathrm{mmol})$ was added to an off white suspension of ligand $\mathbf{1}-\left(\mathbf{P F}_{\mathbf{6}}\right)_{2}(166 \mathrm{mg}, 0.2 \mathrm{mmol})$ in THF. The resultant yellowish brown solution was stirred at RT for $2 \mathrm{~h}$. Subsequently, a pre-cooled THF solution of $\mathrm{UCl}_{4}(76 \mathrm{mg}, 0.2 \mathrm{mmol})$ was added. A yellowish green precipitate formed immediately upon addition of $\mathrm{UCl}_{4}$. The suspension was stirred for another $1 \mathrm{~h}$ and then filtered through Celite and washed with THF $(5 \mathrm{~mL} \times 3)$. The residue was then dissolved in dichloromethane and the resultant yellowish green solution was concentrated to ca. $5 \mathrm{~mL}$ and cooled at $-35^{\circ} \mathrm{C}$ overnight to afford yellow-green crystals. Once obtained as crystalline material, $\mathbf{2}$ is insoluble in aromatic and aliphatic solvents, and consequently a satisfactory ${ }^{13} \mathrm{C}$ NMR spectrum was not available. However, in dichloromethane- $\mathrm{d}_{2}$, complex 2 decomposes slowly, allowing for ${ }^{1} \mathrm{H}$ and ${ }^{31} \mathrm{P}$ NMR spectroscopic measurement. Crystalline yield: $122 \mathrm{mg}$, 66\%. ${ }^{1} \mathrm{H}-\mathrm{NMR}\left(\mathrm{CD}_{2} \mathrm{Cl}_{2}, 298 \mathrm{~K}, 400 \mathrm{MHz}\right): \delta 14.19$ (s), 9.71 (s), 8.79 (s), -7.35 (s), and $-41.54(\mathrm{~s}) \mathrm{ppm} .{ }^{31} \mathrm{P}\left\{{ }^{1} \mathrm{H}\right\}-\mathrm{NMR}\left(\mathrm{CD}_{2} \mathrm{Cl}_{2}, 298 \mathrm{~K}, 162.0 \mathrm{MHz}\right):-215.87(\mathrm{~s})$ ppm. Anal. Calcd. for $\mathrm{C}_{35} \mathrm{H}_{28} \mathrm{Cl}_{4} \mathrm{~N}_{2} \mathrm{P}_{2} \mathrm{U}$ : C 45.77; $\mathrm{H}$ 3.07; N 3.05. Found: C 45.91; $\mathrm{H}$ 3.03; N 3.01. FTIR $v / \mathrm{cm}^{-1}$ (Nujol): 1573 (w), $1438(\mathrm{~s}), 1216(\mathrm{w}), 1106$ (s), 989 (m), $918(\mathrm{w}), 787(\mathrm{w}), 770(\mathrm{w}), 743(\mathrm{~s}), 690(\mathrm{~s}), 561(\mathrm{w}), 530(\mathrm{~m}), 495(\mathrm{~m})$.

Preparation of 4. A white suspension of $3-(\mathbf{B r})_{2}(141 \mathrm{mg}, 0.2 \mathrm{mmol})$ in toluene $(10 \mathrm{~mL})$ was treated with $0.2 \mathrm{~mL}$ NaHMDS $(2 \mathrm{M}$ in THF, $0.4 \mathrm{mmol})$. The resultant yellow suspension was stirred at RT for $2 \mathrm{~h}$ and then filtered. The filtrate was concentrated to ca. $5 \mathrm{~mL}$ and a cold solution of $\mathrm{UCl}_{4}(76 \mathrm{mg}, 0.2 \mathrm{mmol})$ in THF $(5 \mathrm{~mL})$ was added. The resultant mixture was stirred at RT for another $1 \mathrm{~h}$ before filtration, generating a yellowish green suspension. The residue was washed by toluene $(5 \mathrm{~mL} \times 3)$ and then dissolved in DCM. The green filtrate was concentrated and kept at $-35^{\circ} \mathrm{C}$ overnight to afford yellowish green micro crystalline product. The crystals were collected and washed with cold THF to afford complex 4 in 52\% yield ( $95 \mathrm{mg}$ ). Once obtained as crystalline material from DCM, 4 is not soluble in aromatic and aliphatic solvents, and decomposes in polar solvents such as DCM. Alternatively, it was found that $\mathbf{4}$ could also crystalize from a mixture of deprotonated 3 and $\mathrm{UCl}_{4}$ in THF. Thus, the ${ }^{31} \mathrm{P}$ NMR spectrum of 4 was attained by measuring the reaction mixture after $1 \mathrm{~h}$ in THF- $\mathrm{d}_{8}$. However, satisfactory ${ }^{1} \mathrm{H}$ and ${ }^{13} \mathrm{C}$ NMR spectra could not be obtained and a satisfactory electronic absorption spectrum is also not available for the same reason. ${ }^{31} \mathrm{P}\left\{{ }^{1} \mathrm{H}\right\}-\mathrm{NMR}\left(\mathrm{THF}-\mathrm{d}_{8}, 298 \mathrm{~K}\right.$, $162 \mathrm{MHz}): \delta-125.51(\mathrm{~d}, J=2 \mathrm{~Hz}),-155.13 \mathrm{ppm}(\mathrm{d}, J=2.2 \mathrm{~Hz})$. Anal. Calcd. for $\mathrm{C}_{36} \mathrm{H}_{29} \mathrm{Cl}_{4} \mathrm{NP}_{2} \mathrm{U}$ : C 47.13; H 3.19; N 1.53. Found: C 46.74; H 3.51; N 1.23. FTIR v/ $\mathrm{cm}^{-1}$ (Nujol): 1586 (w), 1439 (s), 1109 (m), 996 (w), 918 (s), 741 (s), 687 (m), 542 (w), $510(\mathrm{w}), 497(\mathrm{w})$.

Preparation of 6. A white suspension of 5-(Br $)_{2}(140 \mathrm{mg}, 0.2 \mathrm{mmol})$ in toluene $(10 \mathrm{~mL})$ was treated with $0.2 \mathrm{~mL}$ NaHMDS ( $2 \mathrm{M}$ in THF, $0.4 \mathrm{mmol})$. The resultant yellow suspension was stirred at RT for $2 \mathrm{~h}$ before filtration. The filtrate was concentrated to ca. $5 \mathrm{~mL}$ and a cold solution of $\mathrm{UCl}_{4}(76 \mathrm{mg}, 0.2 \mathrm{mmol})$ in THF $(5 \mathrm{~mL})$ was added. The resultant mixture was stirred at RT for another $1 \mathrm{~h}$, generating white precipitates. The solvent was removed in vacuo and the yellowish green residue was washed with toluene $(5 \mathrm{~mL} \times 3)$ and then dissolved in DCM and filtered. The green filtrate was concentrated and kept at $-35^{\circ} \mathrm{C}$ overnight to afford 
yellowish green microcrystals. Those crystals were collected and washed with cold THF to afford complex 6 in 59\% yield $(109 \mathrm{mg})$. Once obtained as crystalline material from DCM, $\mathbf{6}$ is not soluble in common organic solvent, and decomposes in THF and DCM. Attempts to characterize $\mathbf{6}$ from reaction mixtures in THF- $\mathrm{d}_{8}$ failed to obtain reliable information. Therefore, no spectral is available for complex 6. The reproducibility of the synthesis of complex $\mathbf{6}$ was assessed by solving and refining the data of single crystal collected from three independent syntheses. Those results were identical. Anal. Calcd. for $\mathrm{C}_{37} \mathrm{H}_{30} \mathrm{Cl}_{4} \mathrm{P}_{2} \mathrm{U}$ : C 48.49; $\mathrm{H} 3.30$. Found: C 45.93; H 3.24. This complex consistently has low carbon content, possibly due to the high sensitivity. FTIR $v / \mathrm{cm}^{-1}$ (Nujol): 1438 (s), $1109(\mathrm{~m}), 996(\mathrm{~m}), 918$ (w), $797(\mathrm{w}), 736(\mathrm{w}), 686(\mathrm{~m}), 559(\mathrm{~s}), 542(\mathrm{~m}), 499(\mathrm{w})$.

\section{Data availability}

The X-ray crystallographic coordinates for structures reported in this study have been deposited at the Cambridge Crystallographic Data Centre (CCDC), under deposition numbers CCDC-1850097 (2), 1850102 (4), and 1850103 (6). These data can be obtained free of charge from The Cambridge Crystallographic Data Centre via www.ccdc.cam.ac.uk/data_request/cif. The data that support the findings of this study are available from the corresponding author upon reasonable request.

Received: 25 July 2018 Accepted: 30 October 2018

Published online: 27 November 2018

\section{References}

1. Frey, G. D., Lavallo, V., Donnadieu, B., Schoeller, W. W. \& Bertrand, G. Facile splitting of hydrogen and ammonia by nucleophilic activation at a single carbon center. Science 316, 439-441 (2007).

2. Dyker, C. A. \& Bertrand, G. Chemical bonding: Rethinking carbon. Nat. Chem. 1, 265-266 (2009).

3. Alcarazo, M., Lehman, C. W., Anoop, A., Thiel, W. \& Fürstner, A. Coordination chemistry at carbon. Nat. Chem. 1, 295-301 (2009).

4. Frenking, G. Dative bonds in main-group compounds: a case for more arrows! Angew. Chem. Int. Ed. 53, 6040-6046 (2014).

5. Igau, A., Grützmacher, H., Baceiredo, A. \& Bertrand, G. Analogous $\alpha, \alpha^{\prime}$-biscarbenoid triply bond species: synthesis of a stable $\lambda^{3}$-phosphinocarbene- $\lambda^{5}$ phosphaacetylene. J. Am. Chem. Soc. 110, 6463-6466 (1988).

6. Arduengo, A. J., Harlow, R. L. \& Kline, M. A stable crystalline carbene. J. Am. Chem. Soc. 113, 361-363 (1991).

7. Arduengo, A. J. Looking for stable carbenes: the difficulty in starting anew. Acc. Chem. Res. 32, 913-921 (1999).

8. Hopkinson, M. N., Richter, C., Schedler, M. \& Glorius, F. An overview of Nheterocyclic carbenes. Nature 510, 485-496 (2014).

9. Peris, E. Smart N-heterocyclic carbene ligands in catalysis. Chem. Rev. 118, 9988-10031 (2017)

10. Lavallo, V., Canac, Y., Präsang, C., Donnadieu, B. \& Bertrand, G. Stable cyclic (alkyl)(amino) carbenes as rigid or flexible, bulky, electron-rich ligands for transition-metal catalysts: a quaternary carbon atom makes the difference. Angew. Chem. Int. Ed. 44, 5705-5709 (2005).

11. Melaimi, M., Soleilhavoup, M. \& Bertrand, G. Stable cyclic carbenes and related species beyond diaminocarbenes. Angew. Chem. Int. Ed. 49, 8810-8849 (2010).

12. Soleilhavoup, M. \& Bertrand, G. Cyclic (alkyl)(amino)carbenes (CAACs): stable carbenes on the rise. Acc. Chem. Res. 48, 256-266 (2015).

13. Bertrand, G., Sioleilhavoup, M., Melaimi, M. \& Jazzar, R. Cyclic (alkyl) (amino)carbenes (CAACs): recent developments. Angew. Chem. Int. Ed. 56, 10046-10068 (2017).

14. Tonner, R. \& Frenking, G. C(NHC) $)_{2}$ : Divalent carbon(0) compounds with $\mathrm{N}-$ heterocyclic carbene ligands-theoretical evidence for a class of molecules with promising chemical properties. Angew. Chem. Int. Ed. 46, 8695-8698 (2007).

15. Dyker, C. A., Lavallo, V., Donnadieu, B. \& Bertrand, G. Synthesis of an extremely bent acyclic allene (a "carbodicarbene"): a strong donor ligand. Angew. Chem. Int. Ed. 47, 3206-3209 (2008).

16. Fürstner, A., Alcarazo, M., Goddard, R. \& Lehmann, C. W. Coordination chemistry of ene-1,1-diamines and a prototype "carbodicarbene". Angew. Chem. Int. Ed. 47, 3210-3214 (2008).

17. Kaufhold, O. \& Hahn, F. E. Carbodicarbenes: divalent carbon(0) compounds. Angew. Chem. Int. Ed. 47, 4057-4061 (2008).

18. Lavallo, V., Dyker, C. A., Donnadieu, B. \& Bertrand, G. Synthesis and ligand properties of stable five-membered-ring allenes containing only second-row elements. Angew. Chem. Int. Ed. 47, 5411-5414 (2008).

19. Melaimi, M., Parameswaran, P., Donnadieu, B., Frenking, G. \& Bertrand, G. Synthesis and ligand properties of a persistent, all-carbon four-membered-ring allene. Angew. Chem. Int. Ed. 48, 4792-4795 (2009).
20. Fernández, I. et al. Exocyclic delocalization at the expense of aromaticity in 3,5 -bis( $\pi$-donor) substituted pyrazolium ions and corresponding cyclic bent allenes. J. Am. Chem. Soc. 26, 7287-7291 (2009).

21. Goldfogel, M. J., Roberts, C. C. \& Meek, S. J. Intermolecular hydroamination of 1,3-dienes catalyzed by bis(phosphine) carbodicarbene-rhodium complexes. J. Am. Chem. Soc. 136, 6227-6230 (2014).

22. Roberts, C. C., Matías, D. M., Goldfogel, M. J. \& Meek, S. J. Lewis acid activation of carbodicarbene catalysts for Rh-catalyzed hydroarylation of dienes. J. Am. Chem. Soc. 137, 6488-6491 (2015).

23. Hsu, Y. C. et al. Synthesis and isolation of an acyclic tridentate bis(pyridine) carbodicarbene and studies on its structural implications and reactivities. Angew. Chem. Int. Ed. 54, 2420-2424 (2015).

24. Pranckevicius, C., Liu, L., Bertrand, G. \& Stephan, D. W. Synthesis of a carbodicyclopropenylidene: a carbodicarbene based solely on carbon. Angew. Chem. Int. Ed. 55, 5536-5540 (2016).

25. Chen, W. C. et al. Carbodicarbenes: unexpected $\pi$-accepting ability during reactivity with small molecules. J. Am. Chem. Soc. 139, 12830-12836 (2017).

26. Ramirez, F., Desai, N. B., Hansen, B. \& McKelvie, N. Hexaphenylcarbodiphosphorane, $\left(\mathrm{C}_{6} \mathrm{H}_{5}\right)_{3} \mathrm{PCP}\left(\mathrm{C}_{6} \mathrm{H}_{5}\right)_{3}$. J. Am. Chem. Soc. 83, 3539-3540 (1961)

27. Tonner, R., Öxler, F., Neumüller, B., Petz, W. \& Frenking, G. Carbodiphosphoranes: the chemistry of divalent carbon(0). Angew. Chem. Int. Ed. 45, 8038-8042 (2006).

28. Tonner, R. \& Frenking, G. Divalent carbon(0)chemistry, part 1: parent compounds. Chem. Eur. J. 14, 3260-3272 (2008).

29. Tonner, R. \& Frenking, G. Divalent carbon(0)chemistry, part 2: protonation and complexes with main group and transition metal Lewis acids. Chem. Eur. J. 14, 3273-3289 (2008).

30. Frenking, G. et al. New bonding modes of carbon and heavier group 14 atoms Si-Pb. Chem. Soc. Rev. 43, 5106-5139 (2014).

31. Alcarazo, M., Radkowski, K., Mehler, G., Goddard, R. \& Fürstner, A. Chiral heterobimetallic complexes of carbodiphosphoranes and phosphinidene-carbene adducts. Chem. Commun. 49, 3140-3142 (2013).

32. Petz, W. Addition compounds between carbones, $\mathrm{CL}_{2}$, and main group Lewis acids: a new glance at old and new compounds. Coord. Chem. Rev. 291, 1-27 (2015).

33. Zhao, L., Hermann, M., Holzmann, N. \& Frenking, G. Dative bonding in main group compounds. Coord. Chem. Rev. 344, 163-204 (2017).

34. Sundermeyer, J., Weber, K., Peters, K. \& von Schnering, H. G. Modeling surface reactivity of metal oxides: synthesis and structure of an ionic organorhenyl perrhenate formed by ligand-induced dissociation of covalent $\mathrm{Re}_{2} \mathrm{O}_{7}$. Organometallics 13, 2560-2562 (1994).

35. Inés, B. et al. Synthesis, structure, and reactivity of a dihydrido borenium cation. Angew. Chem. Int. Ed. 50, 8400-8403 (2011).

36. Chi, C. et al. Preparation and characterization of uranium-iron triple-bonded $\mathrm{UFe}(\mathrm{CO})_{3}{ }^{-}$and $\mathrm{OUFe}(\mathrm{CO})_{3}{ }^{-}$complexes. Angew. Chem. Int. Ed. 56, 6932-6936 (2017)

37. Lu, E., Wooles, A. J., Gregson, M., Cobb, P. J. \& Liddle, S. T. A very short uranium(IV)-rhodium(I) bond with net double-dative bonding character. Angew. Chem. Int. Ed. 57, 6587-6591 (2018).

38. Fox, A. R., Bart, S. C., Meyer, K. \& Cummins, C. C. Towards uranium catalysts. Nature 455, 341-349 (2008)

39. Arnold, P. L., Mansell, S. M., Maron, L. \& McKay, D. Spontaneous reduction and C-H borylation of arenes mediated by uranium(III) disproportionation. Nat. Chem. 4, 668-674 (2012).

40. Halter, D. P., Heinemann, F. W., Bachmann, J. \& Meyer, K. Uraniummediated electrocatalytic dihydrogen production from water. Nature 530, 317-321 (2016).

41. Falcone, M., Chatelain, L., Scopelliti, R., Živković, I. \& Mazzanti, M. Nitrogen reduction and functionalization by a multimetallic uranium nitride complex. Nature 547, 332-335 (2017)

42. Arnold, P. L. \& Turner, Z. R. Carbon oxygenate transformations by actinide compounds and catalysts. Nat. Rev. Chem. 1, 0002 (2017).

43. Halter, D. P., Heinemann, F. W., Maron, L. \& Meyer, K. The role of uranium-arene bonding in $\mathrm{H}_{2} \mathrm{O}$ reduction catalysis. Nat. Chem. 10, 259-267 (2018).

44. Hayton, T. W. Metal-ligand multiple bonding in uranium: structure and reactivity. Dalton Trans. 39, 1145-1158 (2010).

45. Hayton, T. W. Recent developments in actinide-ligand multiple bonding. Chem. Commun. 49, 2956-2973 (2013).

46. Gregson, M., Wooles, A. J., Cooper, O. J. \& Liddle, S. T. Covalent uranium carbene chemistry. Comments on Inorganic Chemistry 35, 262-294 (2015).

47. Cramer, R. E., Maynard, R. B., Paw, J. C. \& Gilje, J. W. A uranium-carbon multiple bond. Crystal and molecular structure of $\left(\eta^{5}-\mathrm{C}_{5} \mathrm{H}_{5}\right)_{3} \mathrm{UCHP}$ $\left(\mathrm{CH}_{3}\right)_{2}\left(\mathrm{C}_{6} \mathrm{H}_{5}\right)$. J. Am. Chem. Soc. 103, 3589-3590 (1981).

48. Fortier, S., Walensky, J. R., Wu, G. \& Hayton, T. W. Synthesis of a phosphorano-stabilized U(IV)-carbene via one-electron oxidation of a U(III)ylide adduct. J. Am. Chem. Soc. 133, 6894-6897 (2011). 
49. Tourneux, J. C. et al. Uranium(IV) nucleophilic carbene complexes. Organometallics 30, 2957-2971 (2011).

50. Rungthanaphatsophon, P., Huang, P. \& Walensky, J. R. Phosphoranostabilized carbene complexes with short thorium(IV) - and uranium(IV) - carbon bonds. Organometallics 37, 1884-1891 (2018).

51. Cantat, T. et al. The $\mathrm{U}=\mathrm{C}$ double bond: synthesis and study of uranium nucleophilic carbene complexes. J. Am. Chem. Soc. 131, 963-972 (2009).

52. Tourneux, J. C. et al. Easy access to uranium nucleophilic carbene complexes. Dalton Trans. 39, 2494-2496 (2010).

53. Mills, D. P. et al. A delocalized arene-bridged diuranium single-molecule magnet. Nat. Chem. 3, 454-460 (2011).

54. Cooper, O. J. et al. Uranium-carbon multiple bonding: facile access to the pentavalent uranium carbene $\left[\mathrm{U}\left\{\mathrm{C}\left(\mathrm{PPh}_{2} \mathrm{NSiMe}_{3}\right)_{2}\right\}(\mathrm{Cl})_{2}(\mathrm{I})\right]$ and comparison of $\mathrm{UV}^{\mathrm{V}}=\mathrm{C}$ and $\mathrm{U}^{\mathrm{IV}}=\mathrm{C}$ bonds. Angew. Chem. Int. Ed. 50, 2383-2386 (2011).

55. Tourneux, J. C. et al. Exploring the uranyl organometallic chemistry: from single to double uranium-carbon bonds. J. Am. Chem. Soc. 133, 6162-6165 (2011).

56. Ma, G., Ferguson, M. J., McDonald, R. \& Cavell, R. G. Actinide metals with multiple bonds to carbon: synthesis, characterization, and reactivity of U(IV) and $\mathrm{Th}(\mathrm{IV})$ bis(iminophosphorano) methandiide pincer carbene complexes. Inorg. Chem. 50, 6500-6508 (2011).

57. Mills, D. P. et al. Synthesis of a uranium(VI)-carbene: reductive formation of uranyl( $(\mathrm{V})$-methanides, oxidative preparation of a $\left[\mathrm{R}_{2} \mathrm{C}=\mathrm{U}=\mathrm{O}\right]^{2+}$ analogue of the $[\mathrm{O}=\mathrm{U}=\mathrm{O}]^{2+}$ uranyl ion $\left(\mathrm{R}=\mathrm{Ph}_{2} \mathrm{PNSiMe}_{3}\right)$, and comparison of the nature of $\mathrm{U}^{\mathrm{VV}}=\mathrm{C}, \mathrm{UV}^{\mathrm{V}}=\mathrm{C}$, and $\mathrm{U}^{\mathrm{VI}}=\mathrm{C}$ double bonds. J. Am. Chem. Soc. 134, 10047-10054 (2012).

58. Cooper, O. J. et al. The nature of the $\mathrm{U}=$ Cdouble bond: Pushing the stability of high-oxidation-state uranium carbenes to the limit. Chem. Eur. J. 19, 7071-7083 (2013).

59. Lu, E. et al. Synthesis, characterization, and reactivity of a uranium(VI) carbene imido oxo complex. Angew. Chem. Int. Ed. 53, 6696-6700 (2014).

60. Gregson, M. et al. Emergence of comparable covalency in isostructural cerium (IV)- and uranium(IV)-carbon multiple bonds. Chem. Sci. 7, 3286-3297 (2016).

61. Gregson, M. et al. The inverse-trans-influence in tetravalent lanthanide and actinide bis(carbene) complexes. Nat. Commun. 8, 14137 (2017).

62. Lu, E., Boronski, J. T., Gregson, M., Wooles, A. J. \& Liddle, S. T. Silylphosphino-carbene complexes of uranium(IV). Angew. Chem. Int. Ed. 57, 5506-5511 (2018).

63. Wooles, A. J. et al. Uranium(III)-carbon multiple bonding supported by arene $\delta$-bonding in mixed-valence hexauranium nanometre-scale rings. Nat. Commun. 9, 2097 (2018).

64. Lyon, J. T. \& Andrews, L. Formation and characterization of the uranium methylidene complexes $\mathrm{CH}_{2}=\mathrm{UHX}(\mathrm{X}=\mathrm{F}, \mathrm{Cl}$, and $\mathrm{Br})$. Inorg. Chem. 45, 1847-1852 (2006).

65. Lyon, J. T., Hu, H.-S., Andrews, L. \& Li, J. Formation of unprecedented actinide carbon triple bonds in uranium methylidyne molecules. Proc. Natl Acad. Sci. USA 104, 18919-18924 (2007).

66. Li, J., Hu, H. S., Lyon, J. T. \& Andrews, L. Chirality, agostic interactions, and pyramidality in actinide methylidene complexes. Angew. Chem. Int. Ed. 46, 9045-9049 (2007).

67. Lyon, J. T., Andrews, L., Hu, H. S. \& Li, J. Infrared spectra and electronic structures of agostic uranium methylidene molecules. Inorg. Chem. 47, 1435-1442 (2008).

68. Hu, H. S., Qiu, Y. H., Xiong, X. G., Schwarz, W. E. \& Li, J. On the maximum bond multiplicity of carbon: unusual $\mathrm{C} \equiv \mathrm{U}$ quadruple bonding in molecular CUO. Chem. Sci. 3, 2786-2796 (2012).

69. Cho, H.-G. \& Andrews, L. Matrix preparation and spectroscopic and theoretical investigation of small high oxidation-state complexes of groups 312,14 , lanthanide and actinide metal atoms: carbon-metal single, double and triple bonds. Coord. Chem. Rev. 335, 76-102 (2017).

70. Zhang, $\mathrm{X}$. et al. A diuranium carbide cluster stabilized inside a $\mathrm{C}_{80}$ fullerene cage. Nat. Commun. 9, 2753 (2018).

71. Mao, W., Xiang, L., Maron, L., Leng, X. \& Chen, Y. Nonchelated phosphoniomethylidene complexes of scandium and lutetium. J. Am. Chem. Soc. 139, 17759-17762 (2017).

72. Luo, L., Li, L. \& Marks, T. J. Energetics of metal-ligand multiple bonds. Thermochemistry of tantalum(V) alkylidene formation. J. Am. Chem. Soc. 119, 8574-8575 (1997).
73. Liddle, $S$. T. et al. $\sigma$ and $\pi$ Donation in an unsupported uranium-gallium bond. Angew. Chem. Int. Ed. 48, 1077-1080 (2009).

74. Nalewajski, R. F. \& Mrozek, J. Modified valence indices from the two-particle density matrix. Int. J. Quantum Chem. 51, 187-200 (1994).

75. Nalewajski, R. F., Mrozek, J., Formosinho, S. J. \& Varandas, A. J. C. Quantum mechanical valence study of a bond-breaking-bond-forming process in triatomic systems. Int. J. Quantum Chem. 52, 1153-1176 (1994).

76. Nalewajski, R. F. \& Mrozek, J. Hartree-Fock difference approach to chemical valence: Three-electron indices in UHF approximation. Int. J. Quantum Chem. 57, 377-389 (1996).

77. Michalak, A., DeKock, R. L. \& Ziegler, T. Bond multiplicity in transition-metal complexes: Applications of two-electron valence indices. J. Phys. Chem. A 112, 7256-7263 (2008).

78. Bader, R. F. W., Slee, T. S., Cremer, D. \& Kraka, E. Description of conjugation and hyperconjugation in terms of electron distributions. J. Am. Chem. Soc. 105, 5061-5068 (1983).

\section{Acknowledgements}

This research was supported by the National Natural Science Foundation of China (Grant Nos. 21772088 and 21703099), the Natural Science Foundation of Jiangsu Province (Grant Nos. BK20170635 and BK20170964), the Fundamental Research Funds for the Central Universities, the program of Jiangsu Specially-Appointed Professor, and the Young Elite Scientist Sponsorship Program of China Association of Science and Technology. S.P. thanks Nanjing Tech University for the postdoctoral fellowship and the High Performance Computing Center of Nanjing Tech University for supporting the computational resources. L.Z. and G.F. acknowledge financial support from Nanjing Tech University (Grant Nos. 39837123 and 39837132) and a SICAM Fellowship from Jiangsu National Synergetic Innovation Center for Advanced Materials.

\section{Author contributions}

C.Z., G.F. and L.Z. conceived this project. W.S. and X.S. performed the experiments and solved all the X-ray structures. C.Z. and W.S. analyzed the experimental data. S.P. conducted the theoretical calculations. L.Z. and G.F. analyzed the theoretical results. C.Z., L.Z. and G.F. drafted the paper with support from W.S. and S.W. All authors discussed the results and contributed to the preparation of the final manuscript.

\section{Additional information}

Supplementary Information accompanies this paper at https://doi.org/10.1038/s41467 018-07377-6.

Competing interests: The authors declare no competing interests.

Reprints and permission information is available online at http://npg.nature.com/ reprintsandpermissions/

Publisher's note: Springer Nature remains neutral with regard to jurisdictional claims in published maps and institutional affiliations.

Open Access This article is licensed under a Creative Commons Attribution 4.0 International License, which permits use, sharing, adaptation, distribution and reproduction in any medium or format, as long as you give appropriate credit to the original author(s) and the source, provide a link to the Creative Commons license, and indicate if changes were made. The images or other third party material in this article are included in the article's Creative Commons license, unless indicated otherwise in a credit line to the material. If material is not included in the article's Creative Commons license and your intended use is not permitted by statutory regulation or exceeds the permitted use, you will need to obtain permission directly from the copyrigh holder. To view a copy of this license, visit http://creativecommons.org/licenses/by/4.0/.

(C) The Author(s) 2018 